\title{
Genetic variants in the $B D N F$ gene and therapeutic response to risperidone in schizophrenia patients: a pharmacogenetic study
}

\author{
Mingqing Xü $u^{\star, 1,2,3,4}$, Sheng Li ${ }^{3,4}$, Qinghe Xing ${ }^{3,4}$, Rui Gao ${ }^{3,4}$, Guoyin Feng ${ }^{5}$, Zhiguang Lin $^{5}$, David St Clair ${ }^{6}$ \\ and Lin $\mathrm{He}^{\star, 3,4,7}$
}

Risperidone is a widely used atypical antipsychotic agent that produces considerable interindividual differences in patient response. We investigated the pharmacogenetic relationship between the brain-derived neurotrophic factor (BDNF) gene and response to risperidone in $127 \mathrm{Han}$ Chinese schizophrenic patients. Three functional polymorphisms, (GT) ${ }_{n}$ dinucleotide repeat polymorphism, C-270T, and the rs6265G/A single-nucleotide polymorphism (SNP), were genotyped and analyzed for association, with reduction of Brief Psychiatric Rating Scale (BPRS) scores following an 8-week period of risperidone monotherapy. For individual polymorphic analysis, we found that the frequency of the 230-bp allele of the (GT) polymorphism was much higher in responders $(47.95 \%)$ than in nonresponders $(32.41 \%)$ and the difference was statistically significant even after Bonferroni's adjustment (for the 230-bp allele: adjusted $P=0.039$ ). For haplotype-based analyses of the three polymorphisms, no positive finding was observed in the global test, but in specific haplotype tests, two haplotypes were also significantly related to response to risperidone (for haplotype 230-bp/C-270/rs6265G: $P=0.0009$; for haplotype 234-bp/C-270/rs6265A: $P=0.043$ ), indicating that patients with the 230-bp allele of the $(G T)_{\mathrm{n}}$ polymorphism or the 230-bp/C-270/rs6265G haplotype responded better to risperidone than those with other alleles or haplotypes, and that the positive effect of the individual haplotype $230-\mathrm{bp} / \mathrm{C}-270 / \mathrm{rs} 6265 \mathrm{G}$ was mainly driven by the 230-bp allele. These findings demonstrate that the individual and combinatorial genetic variants in the BDNF gene might have a role in the therapeutic response to risperidone in the Han Chinese population.

European Journal of Human Genetics (2010) 18, 707-712; doi:10.1038/ejhg.2009.238; published online 20 January 2010

Keywords: brain-derived neurotrophic factor; schizophrenia; genetic polymorphism; risperidone

\section{INTRODUCTION}

Risperidone is one of the most widely used second-generation antipsychotic agents for treatment and maintenance therapy of schizophrenia and other related psychotic disorders. It has many clinical advantages over first-generation antipsychotic agents, such as a lower incidence of extrapyramidal symptoms, more frequent clinically significant improvement, a lower risk of relapse, and a favorable effect on both positive and negative symptoms. ${ }^{1,2}$ However, as with other antipsychotics, significant interindividual differences in therapeutic response to risperidone in schizophrenia patients, both regarding therapeutic response and adverse effects, have also been reported. ${ }^{3,4}$ Genetic factors are commonly considered to be the main cause of these interindividual differences. ${ }^{5-10}$

Previous studies have demonstrated that the expression levels of the human brain-derived neurotrophic factor $(B D N F)$ gene in the prefrontal cortex, hippocampus are decreased in schizophrenic patients. ${ }^{11}$ Clinical psychopharmacological and animal model-based experiments have also shown that antipsychotics can regulate the expression of $B D N F .12-16$ Recently, Lee et al ${ }^{16}$ reported that higher plasma $B D N F$ levels might be associated with better response to risperidone treatment in schizophrenia patients. For these reasons, it has been suggested that antipsychotic drug responses of schizophrenic patients vary according to the functionality of the $B D N F$ gene.

The brain-derived neurotrophic factor $(B D N F)$, a gene encoded on human chromosome $11 \mathrm{p} 13$, is a member of the superfamily of neurotrophins, which has a critical role in promoting and modifying growth, differentiation, and survival of neurons in the central nervous system (CNS) ${ }^{17}$ Several polymorphisms of the BDNF gene have been studied for their roles in both the efficacy and adverse effects of antipsychotic treatment. ${ }^{18-28}$ The most frequently reported studies have focused on the following three functional polymorphisms: (1) the dinucleotide repeat polymorphism $(\mathrm{GT})_{\mathrm{n}}$ in the $5^{\prime}$ noncoding region, $1.4 \mathrm{~kb}$ from the transcription starting site; (2) the C-270T polymorphism in the promoter region; and (3) the Val66Met single-nucleotide

${ }^{1}$ School of Public Health, Harvard University, Boston, MA, USA; ${ }^{2}$ Brigham and Women's Hospital, Harvard Medical School, Boston, MA, USA; ${ }^{3}$ Key Laboratory for the Genetics of Developmental and Neuropsychiatric Disorders (Ministry of Education), Bio-X Center, Shanghai Jiao Tong University, Shanghai, PR China; ${ }^{4}$ Institute for Nutritional Sciences, Chinese Academy of Sciences, Shanghai, PR China; ${ }^{5}$ Shanghai Institute of Mental Health, Shanghai, PR China; ${ }^{6}$ Institute of Medical Sciences, Foresterhill, University of Aberdeen, Aberdeen, UK; ${ }^{7}$ Institutes of Biomedical Sciences, Fudan University, Shanghai, PR China

*Correspondence: Dr Mingqing Xu, Department of Medicine, Brigham and Women's Hospital, Harvard Medical School, 75 Francis Street, Boston, MA 02115, USA.

Tel: +1 617 732-5176; Fax: +1 617 732-5764; E-mail: mxu4@rics.bwh.harvard.edu or Lin He, Institutes of Biomedical Sciences, Fudan University, 138 Yixueyuan Road, Shanghai 200032, PR China or Institute for Nutritional Sciences, Shanghai Institute of Biological Sciences, Chinese Academy of Sciences, 294 Taiyuan Road, Shanghai 200031 , PR China.

Tel: +86 21 628-22491; Fax: +86 21 628-22491; E-mail: helin@bio-x.cn

Received 15 October 2009; revised 26 November 2009; accepted 27 November 2009; published online 20 January 2010 
polymorphism (SNP) (NCBI dbSNP ID: rs6265G/A). The $(\mathrm{GT})_{\mathrm{n}}$ dinucleotide repeat polymorphism and the C-270T polymorphism in the $5^{\prime}$ noncoding region of the BDNF gene may influence changes in BDNF expression..$^{14,19,22}$ The Val66Met polymorphism produces a valine-to-methionine substitution at codon 66 (Val66Met), which is in the BDNF coding region, and exerts an effect on intracellular trafficking and activity-dependent secretion of BDNF. ${ }^{17}$

For the $(\mathrm{GT})_{\mathrm{n}}$ dinucleotide repeat polymorphism, Krebs et al ${ }^{19}$ found an excess of the $172-176 \mathrm{bp}$ alleles of the $(\mathrm{GT})_{\mathrm{n}}$ microsatellite repeat polymorphism in schizophrenic patients, with a late age of onset and a better response to antipsychotic treatment in a French Caucasian population. Our previous study demonstrated that the 230-bp allele of the $(\mathrm{GT})_{\mathrm{n}}$ polymorphism exhibits a lower degree of chlorpromazine-induced extrapyramidal syndromes. ${ }^{18}$ For the C-270T polymorphism, one study has been reported but no association with weight gain adverse effects was found. ${ }^{14}$ For the Val66Met polymorphism, Hong et al ${ }^{20}$ found that the Val/Val genotype was more frequent in clozapine-responding patients; Zhang et al ${ }^{25}$ reported that the Val66Met variation affected weight gain in male patients with schizophrenia after long-term antipsychotic treatment. Other studies found no relationships between this functional polymorphism and the treatment efficacy of antipsychotic agents. ${ }^{21,22,27}$ To our knowledge, no studies to date have produced a combinatorial multilocus analysis of the BDNF gene and antipsychotic efficacy, or have they reported association between this gene and response to risperidone in schizophrenia patients.

To evaluate the importance of the BDNF gene in the therapeutic efficacy of risperidone, we performed an association study on the above three polymorphisms and their possible combinatorial effects in a Han Chinese sample of schizophrenia patients after 8 weeks of risperidone monotherapy.

\section{METHODS}

\section{Clinical sample}

Most of the clinical samples used in this study were described in our previous publications. ${ }^{5-10}$ A total of 127 unrelated schizophrenia patients (45 males and 84 females; mean \pm SD age: $36.1 \pm 11.1$ years) were recruited from the Shanghai Mental Health Center on the basis of the following inclusion criteria: (1) had no physical complications or other psychiatric disorders; (2) had no history suggesting that antipsychotic treatment would be ineffective; (3) satisfied the DSM-IV criteria for schizophrenia; and (4) had not received any medication for 4-6 weeks before this study. All subjects gave signed, written informed consent through their responsible relatives before the study. This study was approved by the Ethics Committee of Human Genetic Resource at Shanghai Jiao Tong University.

All patients received risperidone at an initial dose of $2 \mathrm{mg} / \mathrm{day}$, and dosage was gradually increased to $4 \mathrm{mg} /$ day during the first week. The drug dose was maintained until 2 weeks, and then adjusted according to individual tolerance. Medication compliance was closely monitored and confirmed by nursing staff. During the study period, no other drugs were administered, except flunitrazepam for acute insomnia, sennoside for constipation, and biperiden for extrapyramidal syndrome. The Brief Psychiatric Rating Scale (BPRS) ${ }^{29}$ was used to assess clinical response at the beginning and after 8 weeks of risperidone treatment by two fully qualified psychiatrists who were blind to the genotype of the patients. The definitions of a responder based on BPRS always differ across studies. A reduction of at least $20,30,35,40$, or $50 \%$ of the initial BPRS score has been frequently used as a threshold to define response. ${ }^{30-33}$ However, the results of linking analysis between the CGI-I (clinical global impressionimprovement) score and percentage BPRS change from baseline showed that rating of much improved CGI-I (CGI-I=2) corresponded to percentage BPRS reductions of $41 \%$ in this study. Therefore, we considered the following frequently used threshold to define a response: a $>40 \%$ reduction of the BPRS total score at baseline if the definition of response was CGI-I $>2$. The detailed description was given in our previous reports. ${ }^{5,6}$

\section{Experimental procedure}

Blood samples for the determination of risperidone and 9-hydroxyrisperidone levels were drawn at 8 weeks after initiation of risperidone treatment, between 0800 and 0900 hours, approximately $12 \mathrm{~h}$ after the bedtime dose. The plasma levels of risperidone and 9-hydroxyrisperidone were ascertained through a wellestablished high-performance liquid chromatography procedure. ${ }^{34}$

Genomic DNA was extracted from peripheral blood using a standard phenol/ chloroform method. Determination of genotypes was carried out after clinical outcomes assessment and was completed by researchers who were blind to the outcomes of assessment. The $(\mathrm{GT})_{\mathrm{n}}$ dinucleotide repeat polymorphism was genotyped by Megabace 1000 (Amersham Biosciences, Piscataway, NJ, USA), which is a fluorescence-based DNA genotyping system that uses capillary electrophoresis. For the C-270T and Val66Met SNPs, genotyping experiments were performed by direct sequencing using an ABI BigDye terminator Sequencing kit 3.1 (PerkinElmer, Foster City, CA, USA). To ensure that the genotypes obtained were valid, regenotyping $(\mathrm{GT})_{\mathrm{n}}$ dinucleotide repeat polymorphism and reverse-direction resequencing for each of the two SNPs were performed on 30 randomly selected DNA samples. All results of regenotyping were identical to those obtained from the first round of genotyping experiment. All initial genotyping and final validation procedures were carried out independently by two experienced technicians. Detailed information about primers and of the genotyping procedure was described in our previous publications. ${ }^{18,23}$

\section{Statistical analysis}

A $t$-test was used for group comparisons of age, gender, baseline BPRS scores, dosage of risperidone, and plasma levels of risperidone and 9-hydroxyrisperidone. Genotype and allele frequencies were calculated using the software SPSS 15.0 for WINDOWS (SPSS Inc., Chicago, IL, USA). Deviations from HardyWeinberg equilibrium were assessed at http://www.kursus.kvl.dk/shares/vetgen/ Popgen/genetik/applets/kitest.html. Comparisons of genotypic and allele frequencies between different groups were performed using $\chi^{2}$-tests. Odds ratios (OR) and their 95\% confidence intervals (CI) were estimated for the effects of specified alleles. To construct haplotype block, pairwise linkage disequilibrium (LD) statistics, measured by standardized $D^{\prime}$, were estimated using Haploview 4.1. ${ }^{35}$ Haplotype distribution was inferred on the software PHASE2.1, which is implemented by the Bayesian method. ${ }^{36}$ SPSS 15.0 was also used to compare the discrepancies of haplotype frequencies between subgroups on the basis of $\chi^{2}$-tests, and to calculate OR with 95\% CI. Bonferroni's corrections were conducted for multiple comparisons to minimize the possibility of type I errors. The statistical power of the sample size was estimated using the $\mathrm{G}^{\star}$ Power program. ${ }^{37}$ In this study, the $P$-values were two-tailed and significance was set at $P<0.05$.

\section{RESULTS}

Demographic and clinical characteristics of the patients eligible for analysis are shown in Table 1. More details were described in our previous publications. The study group consisted of 127 patients. At the end of the 8 -week treatment period, 73 patients $(57.48 \%)$ showed a decrease in total BPRS scores of $>40 \%$ and were defined as responders, whereas 54 patients were nonresponders. No significant differences were found with respect to gender, age of onset, duration of illness, average dosage of risperidone, average plasma levels of risperidone and 9-hydroxyrisperidone, and the plasma risperidone/ 9-hydroxyrisperidone ratio between responders to risperidone and nonresponders. However, there were significant differences in baseline scores of BPRS between responders and nonresponders, indicating that patients with more severe symptoms at baseline showed more symptom improvement as a result of risperidone treatment, whereas those with less-severe symptoms showed less symptom improvement.

\section{Effects of individual polymorphism on therapeutic response to risperidone}

In this study, all three polymorphisms were successfully genotyped in the 127 schizophrenic patients. No significant deviations from Hardy- 
Table 1 Baseline demographic and clinical characteristics of all schizophrenic patients

\begin{tabular}{lccc}
\hline Patients & $\begin{array}{c}\text { Total } \\
(\mathrm{n}=127)\end{array}$ & $\begin{array}{c}\text { Responders } \\
(\mathrm{n}=73)\end{array}$ & $\begin{array}{c}\text { Nonresponders } \\
(\mathrm{n}=54)\end{array}$ \\
\hline No. of male patients (\%) & $45(35.43)$ & $24(32.88)$ & $21(38.89)$ \\
Age (years) & $36.1 \pm 11.1$ & $36.7 \pm 10.5$ & $35.4 \pm 12.3$ \\
Age of onset (years) & $30.50 \pm 10.42$ & $31.12 \pm 9.8$ & $30.11 \pm 11.3$ \\
Duration of illness (years) & $6.12 \pm 8.52$ & $5.77 \pm 8.98$ & $6.51 \pm 9.43$ \\
Average applied dosage (mg/day) & $3.21 \pm 1.11$ & $3.15 \pm 1.01$ & $3.37 \pm 0.98$ \\
Plasma risperidone level (ng/ml) & $7.40 \pm 6.85$ & $7.31 \pm 6.18$ & $7.98 \pm 6.75$ \\
Plasma 9-hydroxyrisperidone & $23.20 \pm 16.7$ & $22.39 \pm 14.81$ & $24.84 \pm 18.49$ \\
level (ng/ml) & & & \\
Baseline score of BPRS & $42.37 \pm 12.33$ & $46.12 \pm 13.14$ & $37.56 \pm 9.67$ \\
\hline
\end{tabular}

Weinberg equilibrium were found in the distributions of any genotypes across the overall patient sample. Table 2 shows the genotype and allele frequencies of the three polymorphisms, subdivided according to therapeutic responses. Responder and nonresponder groups were compared for genotype and allele frequencies across the three polymorphisms. Results of the allele- and genotype-wise analysis for individual polymorphisms are presented in Table 3.

For the $(\mathrm{GT})_{\mathrm{n}}$ dinucleotide repeat polymorphism, the overall genotype and allele distributions showed no significant difference between responders and nonresponders (for genotype distribution: $\chi^{2}=11.088$, d.f. $=11, P=0.436$; for allele distribution: $\chi^{2}=7.613$, d.f. $=4$, $P=0.107)$. When considering individual alleles, we found that the frequency of the 230-bp allele was higher in responders than in nonresponders and this was statistically significant even after Bonferroni's adjustment for multiple testing (for the 230-bp allele: $\chi^{2}=6.180$, d.f. $=1, P=0.013, \mathrm{OR}=1.921,95 \% \mathrm{CI}=1.145-3.223$; adjusted $P=0.039$; see Table 3 ). The frequency of the 234-bp allele was lower in responders than in nonresponders, although the statistical significance disappeared after Bonferroni's adjustment (for the 234-bp allele: $\chi^{2}=4.032, \quad$ d.f. $=1, \quad P=0.045, \quad \mathrm{OR}=0.596, \quad 95 \% \quad \mathrm{CI}=0.359-0.989$; adjusted $P=0.135$; see Table 3 ), which indicated that patients with the 230-bp allele had a somewhat better response to risperidone, whereas the response of those with the 234-bp allele seemed to be worse. For the C-270T and Val66Met polymorphisms, no significant discrepancies in allele or genotype frequencies were observed (see Table 3). In addition, no significant differences were observed between the different genotypic subgroups of the three BDNF polymorphisms with respect to risperidone dosages or plasma levels of risperidone and 9-hydroxyrisperidone or the plasma risperidone/9-hydroxyrisperidone ratio $(P>0.05)$ (data not shown). These findings suggest that there were no significant drug-genotype interactions in any genotypic subgroup.

Linkage disequilibrium pattern and haplotype analysis

Pairwise LD estimates (measured in terms of $D^{\prime}$ ) showed strong LD between the three polymorphic markers (see Table 4). As haplotype analysis is particularly suited to resolving the problems inherent in association studies based on individual polymorphisms, ${ }^{38}$ we constructed a haplotype block on the basis of pairwise LD patterns and analyzed the common haplotypes to determine whether the polymorphisms would have greater predictive value when analyzed together. The $\chi^{2}$-based statistical $P$-values corresponding to haplotypes are shown in Table 5. Haplotypes with probabilities greater than $3 \%$ accounted for the majority of haplotype diversity (>90\%).

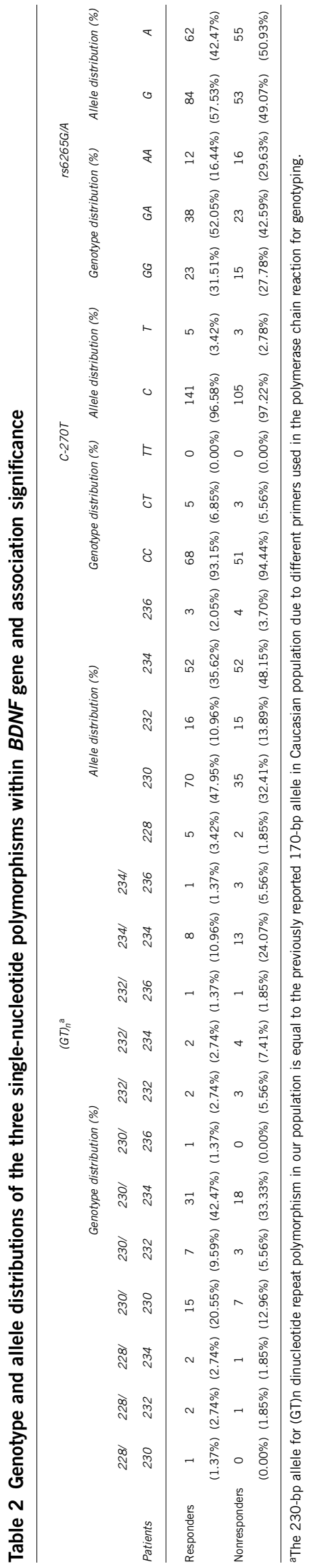


The results indicated that the global frequencies of estimated haplotypes with different combinations of all three polymorphisms were not significantly different in the responder group compared with the nonresponder group $\left(\chi^{2}=7.313\right.$, d.f. $\left.=5, P=0.198\right)$. However, the individual haplotype $230-\mathrm{bp} / \mathrm{C}-270 / \mathrm{rs} 6265 \mathrm{G}$ was positively associated with responders $\left(\chi^{2}=6.901, P=0.0009, \mathrm{OR}=2.014,95 \% \mathrm{CI}=1.190-3.408\right.$; see Table 5), and haplotype $234-\mathrm{bp} / \mathrm{C}-270 / \mathrm{rs} 6265 \mathrm{~A}$ was associated with nonresponders $\left(\chi^{2}=4.091, P=0.043, \mathrm{OR}=0.0584,95 \% \mathrm{CI}=0.347-0.986\right.$; see Table 5).

\section{Evaluation of statistical power}

Power calculations revealed that the sample size recruited for the study had greater than $80 \%$ power in detecting a significant association $(Z<0.05)$ for both allele-based and haplotype-based analyses, given a predefined effective size index of 0.2 (corresponding to a 'weak' genetic effect). This indicates that the sample size in our study was sufficient to achieve a considerably low risk for type II error.

Table 3 Genotype- and allele-wise association analysis

\begin{tabular}{|c|c|c|c|c|c|c|}
\hline \multirow[b]{2}{*}{$\begin{array}{l}\text { Polymorphic } \\
\text { markers }\end{array}$} & \multicolumn{3}{|c|}{ Genotype-wise analysis } & \multicolumn{3}{|c|}{ Allele-wise analysis } \\
\hline & $\chi^{2}$ & $\begin{array}{l}\text { Degree of } \\
\text { freedom }\end{array}$ & P-value & $\chi^{2}$ & $\begin{array}{c}\text { Degree of } \\
\text { freedom }\end{array}$ & P-value \\
\hline$(\mathrm{GT})_{\mathrm{n}}{ }^{\mathrm{a}}$ & 11.088 & 11 & 0.436 & 7.613 & 4 & 0.107 \\
\hline $\mathrm{C}-270 \mathrm{~T}^{\mathrm{b}}$ & 0.133 & 2 & 0.936 & 0.085 & 1 & 0.770 \\
\hline rs6265G/Ac & 3.173 & 2 & 0.205 & 1.788 & 1 & 0.181 \\
\hline
\end{tabular}

${ }^{a}$ For allele-wide analysis of $(\mathrm{GT})_{\mathrm{n}}$ dinucleotide repeat polymorphism, when 230-bp allele was prespecified as response allele: $\chi^{2}=6.180$, degree of freedom $=1, P=0.013$, odds ratio $(95 \%$ confidence interval $)=1.921$ (1.145-3.223); when 232-bp allele was prespecified as response allele: $\chi^{2}=0.497$, degree of freedom $=1, P=0.481$, odds ratio ( $95 \%$ confidence interval $)=0.763(0.359-1.620)$; when 234-bp allele was prespecified as response allele: 2 $\chi^{2}=4.032$, degre

${ }^{\mathrm{b}}$ For allele-wide analysis of C-270T polymorphism, C allele was prespecified as response allele, odds ratio $(95 \%$ confidence interval $)=0.806(0.188-3.447)$

cFor allele-wide analysis of $\mathrm{rs} 6265 \mathrm{G} / \mathrm{A}$ polymorphism, $\mathrm{G}$ allele was prespecified as response allele, odds ratio ( $95 \%$ confidence interval) $=1.406(0.853-2.318)$.

Table 4 Pairwise polymorphisms linkage disequilibrium statistics among the three polymorphic markers

\begin{tabular}{lccc}
\hline Polymorphic markers & $(G T)_{n}$ & C-270T & rs6265G/A \\
\hline$(\mathrm{GT})_{\mathrm{n}}$ & - & & \\
C-270T & 0.92 & - & - \\
rs6265G/A & 0.87 & 0.99 & - \\
\hline
\end{tabular}

If $D^{\prime}>0.6$, the two markers are considered to be in a strong linkage disequilibrium. -: not applicable.

\section{DISCUSSION}

This study has attempted to identify the genetic contribution of the $B D N F$ gene to clinical variability in therapeutic responses to risperidone. However, the factors underlying interindividual variation in response to medication may also include socioeconomic, demographic, clinical, and other environmental variables that may cause interindividual pharmacoepigenetic differences, ${ }^{39}$ and these will probably confound most pharmacogenetic association results. Our study was designed to minimize the effects of such potentially confounding factors. First, the patients involved were receiving atypical antipsychotics for the first time. Previous drug treatment may produce confounding effects on clinical response to subsequent antipsychotic medication. Second, some confounding factors were well matched between subgroups at the time of the study and we also, subsequently, estimated the effects of other potential confounding factors (such as age, age of onset, gender, plasma drug concentrations, initial severity of illness) using the $t$-test.

In our study, three functional genetic polymorphisms in the BDNF gene were investigated for their possible association with risperidone response in 127 unrelated schizophrenic Chinese patients from Shanghai. We found that the frequency of the 230-bp allele of the $(\mathrm{GT})_{\mathrm{n}}$ dinucleotide repeat polymorphism was much higher in responders than in nonresponders and that the difference was statistically significant even after Bonferroni's adjustment for multiple testing; we also found that two haplotypes constructed with the three polymorphisms were significantly related to the response to risperidone, which implied that patients with the 230-bp allele of the $(\mathrm{GT})_{\mathrm{n}}$ dinucleotide repeat polymorphism or the 230-bp/C-270/rs6265G haplotype had better response to risperidone than those with other alleles or haplotypes, especially those with the 234-bp allele and the 234-bp/ C-270/rs6265A haplotype. These findings were consistent with the roles of 230 and 234-bp alleles of the $(\mathrm{GT})_{\mathrm{n}}$ dinucleotide repeat polymorphism in the therapeutic response to risperidone, which indicates that the effects of haplotypes were mainly driven by the $(\mathrm{GT})_{\mathrm{n}}$ dinucleotide repeat polymorphism and that genotyping of the dinucleotide repeat polymorphism is sufficient to assess the major influence of $B D N F$ on response. The 230-bp allele in our study and the 170 -bp allele in the study by Krebs et $a l^{19}$ are of the same allele with the same number of dinucleotide repeats. Both studies indicated that less number of dinucleotide repeats were associated with better response to antipsychotics. However, the dosage effects of the number of dinucleotide repeats on therapeutic response to risperidone were not observed because of the sparse allele distribution of the $(\mathrm{GT})_{\mathrm{n}}$ polymorphism.

Power estimation showed that the sample size in our study was sufficient to achieve a low risk of a type II error under the predefined weak-to-moderate effect size. Our results indicate that the role of

Table 5 Estimated haplotype frequencies and association significance

\begin{tabular}{|c|c|c|c|c|c|c|c|c|}
\hline \multirow[b]{2}{*}{ Haplotype } & \multirow[b]{2}{*}{$(G T)_{n}$} & \multirow[b]{2}{*}{$C-270 T$} & \multicolumn{4}{|c|}{ Frequency (\%) } & \multirow[b]{2}{*}{$\mathrm{P}$-value } & \multirow[b]{2}{*}{ Odds ratio $(95 \% \mathrm{Cl})$} \\
\hline & & & rs6265G/A & Responders & Nonresponders & $\chi^{2}$ & & \\
\hline 1 & 230 & C & G & 45.890 & 29.630 & 6.901 & 0.009 & $2.014(1.190-3.408)$ \\
\hline 2 & 234 & C & $A$ & 29.452 & 41.667 & 4.091 & 0.043 & $0.584(0.347-0.986)$ \\
\hline 3 & 234 & C & $\mathrm{G}$ & 4.795 & 6.250 & 0.339 & 0.560 & $0.727(0.247-2.137)$ \\
\hline 4 & 232 & C & $\mathrm{G}$ & 5.833 & 7.917 & 0.444 & 0.505 & $0.723(0.277-1.886)$ \\
\hline 5 & 232 & $\mathrm{C}$ & $A$ & 5.417 & 4.167 & 0.092 & 0.761 & $1.194(0.380-3.757)$ \\
\hline Global & & & & & & 7.313 & 0.198 & \\
\hline
\end{tabular}

Haplotypes with a frequency lower than $3 \%$ were omitted from analysis. 
individual polymorphisms in the $B D N F$ gene may be weak, but that the combinatorial effects of variants on therapeutic response to risperidone might be moderate. The potential mechanism might be that risperidone can mediate allele-specific gene regulation by modifying the allele functionality for the $(\mathrm{GT})_{\mathrm{n}}$ dinucleotide repeat polymorphism or multiple independent genetic variants in the $B D N F$ gene locus.

Three questions that are beyond the scope of the current study need to be addressed in further studies to comprehensively and systematically understand the pharmacogenetic and biological roles of the $B D N F$ gene in risperidone-treated schizophrenia patients. First, shortterm antipsychotic treatment studies are more likely to be affected by nonspecific confounding factors, such as drug tolerance and adverse effects, than are long-term treatment studies. ${ }^{8,11,13,14}$ The assumed predictive effect of polymorphisms of $B D N F$ for long-term risperidone treatment outcomes should therefore be further evaluated. Further studies on larger groups and on the effects of longer-term antipsychotic treatment are also needed to confirm the present results. Second, the causal relationships between genetic variation and differential gene expression levels and between therapeutic response and the changes in gene expression levels need to be elucidated further. There is no clear evidence to demonstrate that risperidone-mediated expression changes in the BDNF gene are modified by its genetic variants, even though some studies have shown that long-term and high-dose risperidone treatment may significantly downregulate hippocampal BDNF mRNA levels in schizophrenia patients. ${ }^{11,13,14}$ Third, BDNF is a critical regulation factor for the expression of dopamine $\mathrm{D}_{3}(D R D 3)$ in neurodevelopment, ${ }^{40}$ whereas $D R D 3$ is an important gene in the dopaminergic system and acts as an antipsychotic drug target. In addition, BDNF promotes the development and function of serotonergic neurons and serotonergic transmission exerts powerful control over BDNF expression. ${ }^{41} B D N F, D R D 3$, serotonin transporter (SLC6A4), and other genetic and environmental factors involved in neurodevelopment may interact with each other to affect many clinical features in schizophrenia. ${ }^{42}$ Exploring the epitastic interactions between $B D N F$ and serotoninergic and dopaminergic-related genes or among the $B D N F$-mediated pathways is needed to fully understand the role of $B D N F$ in the therapeutic efficacy of risperidone.

In summary, we performed a well-designed association study of the $B D N F$ gene with risperidone response, and discovered that there was significant variability in allele frequencies for the $(\mathrm{GT})_{\mathrm{n}}$ dinucleotide repeat polymorphism between responder and nonresponder groups after Bonferroni's adjustment for multiple testing and that two haplotypes constructed with the three polymorphisms were significantly related to response to risperidone. These results demonstrate that the individual and combinatorial genetic variants in the BDNF gene might have a weak-to-moderate role in affecting therapeutic response to risperidone in the Han Chinese population. The assumed predictive role of polymorphic markers within the BDNF gene for risperidone treatment efficacy should be further evaluated by investigating a set of variants from different genes that might exert a combinatorial or synergistic effect on drug action, as risperidone has also been found to interact with serotoninergic and dopaminergic agents.

\section{CONFLICT OF INTEREST}

The authors declare no conflict of interest.

\section{ACKNOWLEDGEMENTS}

This work was supported by grants (2006AA02A407, 2006CB910601, 2006BAI05A05, 2007CB947300, and 07DZ22917), by the Shanghai Leading
Academic Discipline Project (B205), and by the Shanghai Municipality Science \& Technology Commission (05JC14090). We are grateful to all participants and to the psychiatrists and mental health workers who participated in this project.

1 Csernansky JG, Mahmoud R, Brenner R: Risperidone-USA-79 Study Group. A comparison of risperidone and haloperidol for the prevention of relapse in patients with schizophrenia. N Engl J Med 2002; 346: 16-22.

2 Leucht S, Corves C, Arbter D, Engel RR, Li C, Davis JM: Second-generation versus first-generation antipsychotic drugs for schizophrenia: a meta-analysis. Lancet 2009; 373: 31-41.

3 Lane HY, Liu YC, Huang CL et al: Risperidone-related weight gain: genetic and nongenetic predictors. J Clin Psychopharmacol 2006; 26: 128-134.

4 Stroup TS: Heterogeneity of treatment effects in schizophrenia. Am J Med 2007; 120 (4 Suppl 1): S26-S31.

5 Xing $\mathrm{Q}$, Gao R, Li $\mathrm{H}$ et al: Polymorphisms of the ABCB1 gene are associated with the therapeutic response to risperidone in Chinese schizophrenia patients. Pharmacogenomics 2006; 7: 987-993.

6 Xing Q, Qian X, Li H et al: The relationship between the therapeutic response to risperidone and the dopamine D2 receptor polymorphism in Chinese schizophrenia patients. Int J Neuropsychopharmacol 2007; 10: 631-637.

7 Wang L, Yu L, He G et al: Response of risperidone treatment may be associated with polymorphisms of HTT gene in Chinese schizophrenia patients. Neurosci Lett 2007; 414: $1-4$.

8 Zhang A, Xing Q, Wang L et al: Dopamine transporter polymorphisms and risperidone response in Chinese schizophrenia patients: an association study. Pharmacogenomics 2007; 8: 1337-1345.

9 Xuan J, Zhao X, He G et al: Effects of the dopamine D3 receptor (DRD3) gene polymorphisms on risperidone response: a pharmacogenetic study. Neuropsychopharmacology 2008; 33: 305-311.

10 Gu B, Wang L, Zhang AP et al: Association between a polymorphism of the HTR3A gene and therapeutic response to risperidone treatment in drug-naive Chinese schizophrenia patients. Pharmacogenet Genomics 2008; 18: 721-727.

11 Zhang XY, Zhou DF, Wu GY et al: BDNF levels and genotype are associated with antipsychotic-induced weight gain in patients with chronic schizophrenia. Neuropsychopharmacology 2008; 33: 2200-2205.

12 Chlan-Fourney J, Ashe P, Nylen K, Juorio AV, Li XM: Differential regulation of hippocampal BDNF mRNA by typical and atypical antipsychotic administration. Brain Res 2002; 954: 11-20.

13 Pillai A, Terry Jr AV, Mahadik SP: Differential effects of long-term treatment with typical and atypical antipsychotics on NGF and BDNF levels in rat striatum and hippocampus. Schizophr Res 2006; 82: 95-106.

14 Zhang XY, Tan YL, Zhou DF et al: Serum BDNF levels and weight gain in schizophrenic patients on long-term treatment with antipsychotics. J Psychiatr Res 2007; 41: 997-1004.

15 Rizos EN, Papadopoulou A, Laskos E et al: Reduced serum BDNF levels in patients with chronic schizophrenic disorder in relapse, who were treated with typical or atypical antipsychotics. World J Biol Psychiatry 2008; 10: 1-5.

16 Lee BH, Kim YK: Increased plasma brain-derived neurotropic factor, not nerve growth factor-Beta, in schizophrenia patients with better response to risperidone treatment. Neuropsychobiology 2009; 59: 51-58.

17 Egan MF, Kojima M, Callicott JH et al: The BDNF val66met polymorphism affects activity-dependent secretion of BDNF and human memory and hippocampal function. Cell 2003; 112: 257-269.

$18 \mathrm{Xu} \mathrm{MQ}$, St Clair D, Feng GY et al: BDNF gene is a genetic risk factor for schizophrenia and is related to the chlorpromazine-induced extrapyramidal syndrome in the Chinese population. Pharmacogenet Genomics 2008; 18: 449-457.

19 Krebs MO, Guillin O, Bourdell MC et al: Brain derived neurotrophic factor (BDNF) gene variants association with age at onset and therapeutic response in schizophrenia. Mol Psychiatry 2000; 5: 558-562.

20 Hong CJ, Yu YW, Lin CH, Tsai SJ: An association study of a brain-derived neurotrophic factor Val66Met polymorphism and clozapine response of schizophrenic patients. Neurosci Lett 2003; 349: 206-208.

21 Liou YJ, Liao DL, Chen JY et al: Association analysis of the dopamine D3 receptor gene ser9gly and brain-derived neurotrophic factor gene val66met polymorphisms with antipsychotic-induced persistent tardive dyskinesia and clinical expression in Chinese schizophrenic patients. Neuromolecular Med 2004; 5: 243-251.

22 Anttila S, Illi A, Kampman O, Mattila KM, Lehtimäki T, Leinonen E: Lack of association between two polymorphisms of brain-derived neurotrophic factor and response to typical neuroleptics. J Neural Transm 2005; 112: 885-890.

$23 \mathrm{Xu} \mathrm{MQ}$, St Clair D, Ott J, Feng GY, He L: Brain-derived neurotrophic factor gene C-270T and Val66Met functional polymorphisms and risk of schizophrenia: a moderate-scale population-based study and meta-analysis. Schizophr Res 2007; 91: 6-13.

24 Chao HM, Kao HT, Porton B: BDNF Val66Met variant and age of onset in schizophrenia. Am J Med Genet B Neuropsychiatr Genet 2008; 147: 505-506. 
25 Zhang XY, Zhou DF, Wu GY et al: BDNF levels and genotype are associated with antipsychotic-induced weight gain in patients with chronic schizophrenia. Neuropsychopharmacology 2008; 33: 2200-2205.

26 Ho BC, Andreasen NC, Dawson JD, Wassink TH: Association between brain-derived neurotrophic factor Val66Met gene polymorphism and progressive brain volume changes in schizophrenia. Am J Psychiatry 2007; 164: 1890-1899.

27 Kang SG, Choi JE, An H et al: No association between the brain-derived neurotrophic factor gene Val66Met polymorphism and tardive dyskinesia in schizophrenic patients. Prog Neuropsychopharmacol Biol Psychiatry 2008; 32: 1545-1548.

28 Rybakowski JK: BDNF gene: functional Val66Met polymorphism in mood disorders and schizophrenia. Pharmacogenomics 2008; 9: 1589-1593.

29 Overall JE, Gorham DR: The brief psychiatric rating scale. Psychol Rep 1962; 10: 799-812.

30 Lachar D, Bailley SE, Rhoades HM, Varner RV: Use of BPRS-A percent change scores to identify significant clinical improvement: accuracy of treatment response classification in acute psychiatric inpatients. Psychiatry Res 1999; 89: 259-268.

31 Kumra S, Kranzler H, Gerbino-Rosen G et al: Clozapine and 'high-dose' olanzapine in refractory early-onset schizophrenia: a 12-week randomized and double-blind comparison. Biol Psychiatry 2008; 63: 524-529.

32 Zalsman G, Frisch A, Lev-Ran S et al: DRD4 exon III polymorphism and response to risperidone in Israeli adolescents with schizophrenia: a pilot pharmacogenetic study. Eur Neuropsychopharmacol 2003; 13: 183-185.

33 Sakumoto N, Kondo T, Mihara K, Suzuki A, Yasui-Furukori N: Dopamine D2 receptor gene polymorphisms predict well the response to dopamine antagonists at therapeutic dosages in patients with schizophrenia. Psychiatry Clin Neurosci 2007; 61: 174-180.

34 LLerena A, Berecz $\mathrm{R}$, Dorado $\mathrm{P}$ et al: Determination of risperidone and 9-hydroxyrisperidone in human plasma by liquid chromatography: application to the evaluation of CYP2D6 drug interactions. J Chromatogr B Analyt Technol Biomed Life Sci 2003; 783: 213-219.

35 Barrett JC, Fry B, Maller J, Daly MJ: Haploview: analysis and visualization of LD and haplotype maps. Bioinformatics 2005; 21: 263-265.

36 Stephens M, Smith NJ, Donnelly P: A new statistical method for haplotype reconstruction from population data. Am J Hum Genet 2001; 68: 978-989.

37 Erdfelder E, Faul F, Buchner A: *Power: a general power analysis program. Behav Res Methods Instrum Comput 1996; 28: 1-11.

38 Becker T, Knapp M: A powerful strategy to account for multiple testing in the context of haplotype analysis. Am J Hum Genet 2004; 75: 561-570.

39 Gomez A, Ingelman-Sundberg M: Pharmacoepigenetics: its role in interindividual differences in drug response. Clin Pharmacol Ther 2009; 85: 426-430.

40 Guillin O, Diaz J, Carroll P, Griffon N, Schwartz JC, Sokoloff P: BDNF controls dopamine D3 receptor expression and triggers behavioural sensitization. Nature 2001; 411: 86-89.

41 Martinowich K, Lu B: Interaction between BDNF and serotonin: role in mood disorders. Neuropsychopharmacology 2008; 33: 73-83.

42 Gourion D, Goldberger C, Leroy S, Bourdel MC, Olié JP, Krebs MO: Age at onset of schizophrenia: interaction between brain-derived neurotrophic factor and dopamine D3 receptor gene variants. Neuroreport 2005; 16: 1407-1410. 